\title{
Hausärztliche Highlights vom Deutschen Schmerzkongress
}

\section{STUDIE BEI RÜCKENSCHMERZEN ZEIGT}

\section{Placebo kann analgetisch wirken}

Placebo kann ein hochwirksames Medikament bei Rückenschmerzen sein. Das fanden Forscher des Klinikums Dortmund heraus. Sie verabreichten Patienten mit schweren chronischen Rückenschmerzen ein Placebo, das sie entweder offen als solches deklarierten oder als Opioid ausgaben. Dann ließ man die Patienten die Schmerzbelastung bei Aktivitäten des täglichen Lebens dokumentieren. Wie zu erwarten ergab sich unter dem offenbaren Placebo kaum eine Wirkung. Das als Opioid deklarierte Placebo dagegen hatte eine große Effektstärke. Die Schmerzbelastung verringerte sich und die Aktivitäten konnten angstfreier durchgeführt werden.

Es zeigt sich also, dass allein die Information, ein starkes Schmerzmittel zu be-

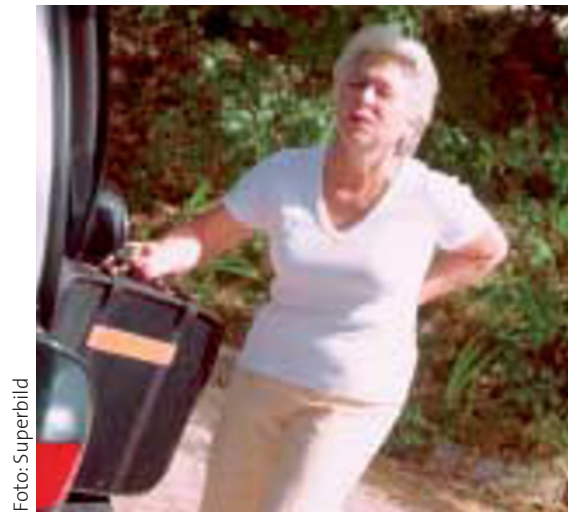

Wie gut eine Schmerztherapie wirkt, hängt auch von der Erwartungshaltung ab.

kommen, zu einem klinisch relevanten analgetischen Effekt führt. Die Frage, ob eine Therapie mit Placebo ethisch zu vertreten ist, bleibt allerdings offen.

\section{DURCH FALLPAUSCHALEN OFT NICHT ABGEDECKT}

\section{Kopfschmerzen stationär abklären?}

Die Aufnahme zur Abklärung von chronischen Kopfschmerzen kann das Krankenhaus teuer zu stehen kommen. Die entsprechenden DRGs decken in vielen Fällen nicht die Kosten ab. K. H. Grotemeyer vom Klinikum Saarbrücken verweist darauf, dass besonders die „Schnellabklärung" in weniger als zwei Tagen unrentabel ist. Ganz besonders dann, wenn ein MRT anfällt. In $64 \%$ der Fälle kam in
Saarbrücken nämlich bei dieser Abklärung nur „Spannungskopfschmerz" heraus. Der wird aber nicht ausreichend vergütet. Dabei war die Verdachtsdiagnose, die die Patienten ins Krankenhaus brachte, oft gravierend. Bei $25 \%$ der Patienten bestand Verdacht auf eine Meningitis, bei 35\% auf eine zerebrale Ischämie oder eine Hirnblutung. Aber das spielt im Nachhinein bei der Abrechnung keine Rolle mehr.

\section{SCHMERZGEDÄCHTNIS LIEGT IM LIMBISCHEN SYSTEM}

\section{Depression als Komorbidität}

Dass Schmerzpatienten zu depressiven Verstimmungen neigen, ist nicht nur ein psychischer Effekt. Wie sich zunehmend zeigt, ist dies eine erlernte und im Gehirn fixierte Reaktion. Offenbar kommt es zu veränderten neuronalen Schaltungen. Diese formen das "Schmerzgedächtnis“: Im limbischen System des Gehirns gibt es neuronale Lernvorgänge, die Schmerzerfahrungen fixieren. Das limbische System ist allerdings auch für unseren emotio- nalen Haushalt zuständig. Auf die chronische Schmerzerfahrung reagiert es, indem es die Emotionen auf Depression schaltet. Neuere Therapiekonzepte gehen davon aus, dass dieses "Schmerzgedächtnis" gelöscht werden muss. Dazu muss der Patient kontinuierlich schmerzfrei sein, damit er seine Schmerzkonditionierung aktiv verlernt.

- Quelle: Deutscher Schmerzkongress, Berlin 24.-27. Oktober 2007
PALLIATIVMEDIZIN

\section{Noch immer zu wenige Betten}

Die Zahl der palliativmedizinischen Betten in Deutschland hat sich seit den 1990er-Jahren fast verzehnfacht. Das klingt viel, ist aber immer noch viel zu wenig. Etwa 2500 Betten stehen derzeit in Deutschland zur Verfügung. Nach britischen Berechnungen sollte aber eine Bettendichte von 50-6o pro einer Million Einwohner vorhanden sein. Der reale Bedarf liegt also bei 5000 Betten. Palliativmediziner hoffen nun auf eine bessere Finanzierung durch die 2007 beschlossene Gesundheitsreform.

\section{MIGRÄNE}

\section{Mit Foramen-ovale- Verschluss kurieren?}

In Reihenuntersuchungen zeigte sich, dass bis zu $80 \%$ der Migränepatienten ein offenes Foramen ovale haben. In der übrigen Bevölkerung liegt die Rate bei circa $20 \%$. In einer prospektiven Studie wurde geprüft, ob ein Verschluss des Foramens die Migränebeschwerden beeinflusst. Tatsächlich reduzierte sich danach die Anfallsfrequenz. Ganz verschwand die Migräne aber nicht. Man vermutet Mikroembolien aus dem Vorhof, die als Migräneauslöser wirken könnten.

\section{RESTLESS-LEGS-SYNDROM}

\section{L-Dopa kann Symptome verschlechtern}

Neurophysiologen der Uni Göttingen weisen darauf hin, dass eine L-Dopa-Therapie beim Restless-Legs-Syndrom die Symptome verstärken kann. Es kann sogar dazu kommen, dass die Symptome dann statt nachts hauptsächlich tagsüber auftreten. In diesem Fall kann es sinnvoll sein, den Patienten auf einen Dopaminagonisten umzustellen. (Die Deutsche Gesellschaft für Neurologie hat Leitlinien zur RLS-Therapie entwickelt. Im Internet unter: www.dgn.org.)

DR. MED. HORST Gross 